\title{
Recognition of Injury Patterns in Transient Lateral Patellar Dislocation on Magnetic Resonance Imaging
}

\author{
Vijinder Arora ${ }^{1}$, Abhiraj Kakkar ${ }^{2}$
}

\begin{abstract}
Aims and Objectives: To determine the prevalence of various injury patterns in patient with transient lateral patellar dislocation (TLPD) on magnetic resonance imaging (MRI).

Materials and methods: Thirty patients with clinical history pointing toward lateral patellar dislocation (LPD) were evaluated for the characteristic associated injury patterns including lateral femoral contusion, medial patellar contusion/osteochondral injury, medial retinaculum/medial patellofemoral ligament (MR/MPFL) injury, significant joint effusion, intraarticular loose bodies, medial collateral ligament (MCL) injury, and medial meniscus tear. Prevalence of all these findings was assessed.

Results: Our study showed some commonly observed injury patterns in patients with TLPD. Of 30 patients, 25 (83.3\%) had MR/MPFL tear in general. Fifty percentage ( 15 of 30 patients) showed MPFL tear at its patellar insertion, $16.6 \%$ ( 5 of 30 patients) at its mid-part, and $46.6 \%$ ( 14 of 30 patients) at its femoral insertion; $80 \%$ (24 of 30 patients) revealed contusions of the lateral femoral condyle, and $73.33 \%$ ( 22 of 30 patients) had contusion/osteochondral injury of medial patella. Other MRI findings in TLPD included significant joint effusion (20 [66.6\%] of 30), patellar tilt (17 [56.6\%] of 30), patellar subluxation (17 [56.6\%] of 30), medial meniscal tear (7 [23.3\%] of 30), medial collateral injury (3 [10\%] of 30), and intraarticular bodies (2 [6.6\%] of 30).

Conclusion: Injury to the MR/MPFL, bony contusion involving lateral femoral condyle, and bony contusion/osteochondral injury involving medial aspect of patella are frequently associated with TLPD. Therefore, these findings should be used to diagnose TLPD.

Clinical significance: These findings can reliably help in diagnosing TLPD, and it is essential for the radiologist to be aware of these so as not to miss the diagnosis.

Keywords: Medial patellofemoral ligament, Osteochondral injury, Transient lateral patellar dislocation (TLPD).

AMEI's Current Trends in Diagnosis \& Treatment (2021): 10.5005/jp-journals-10055-0117
\end{abstract}

\section{INTRODUCTION}

Lateral patellar dislocation (LPD) is among the most common knee injuries in young active adolescents. ${ }^{1,2}$ It has been reported in many studies that with increasing age, the risk of LPD decreases and it becomes negligible after the age of 40 years.,4

In most cases, there is a transient episode of LPD with reduction of dislocated patella on subsequent knee extension. ${ }^{5}$ Therefore, the term transient lateral patellar dislocation (TLPD) is more frequently used. By the time magnetic resonance imaging (MRI) is performed, the patella has usually reduced back to its original position and it is only the secondary signs that aid in the diagnosis.

Clinically, patients present with pain involving medial aspect of anterior compartment of knee, which can be explained by bony or cartilaginous injury to the medial patella and lateral femoral condyle and injuries involving medial retinaculum/medial patellofemoral ligament (MR/MPFL).

The study is intended to illustrate the various patterns of injury in patient with TLPD on MRI.

The patellofemoral joint is formed by articulation of femoral trochlea and patella. TLPD is common due to the diverging pull of the patellar tendons and quadriceps (leading to the formation of $\mathrm{Q}$ angle, measuring approximately $15^{\circ}$ ), which is further worsened by the normal valgus configuration of the resting knee. ${ }^{6-9}$ Patellofemoral joint is stabilized by active stabilizers (extensor muscles) and passive stabilizers (ligaments and bones). MR/MPFL is the most important ligamentous stabilizer. Vastus medialis obliquus counteracts the action of the vastus lateralis and is the most vital active stabilizer. The femoral sulcus

\footnotetext{
1,2Department of Radiodiagnosis and Imaging, Sri Guru Ram Das Institute of Medical Sciences and Research, Amritsar, Punjab, India

Corresponding Author: Vijinder Arora, Department of Radiodiagnosis and Imaging, Sri Guru Ram Das Institute of Medical Sciences and Research, Amritsar, Punjab, India, Phone: +91 7973561408, e-mail: drvijinderarora338@gmail.com

How to cite this article: Arora V, Kakkar A. Recognition of Injury Patterns in Transient Lateral Patellar Dislocation on Magnetic Resonance Imaging. AMEI's Curr Trends Diagn Treat 2021;5(1):1-5.

Source of support: Nil

Conflict of interest: None
}

should be of adequate depth, and the lateral trochlea must be of appropriate height to enable safe tracking throughout the range of patellofemoral flexion. ${ }^{10,11}$

In trochlear dysplasia, the trochlear groove is of suboptimal depth, so it is unable to engage patella especially during early flexion along the knee joint. ${ }^{10}$ Similarly in patella alta, there is reduction in contact between patella and femoral trochlea during early phase of knee flexion, which leads to patellar dislocation. $8,10,12,13$

The characteristic way in which bruising occurs in the lateral femoral condyle and medial patella as mentioned above could easily be understood if we consider the mechanism by which patellar dislocation occurs. The injury mostly occurs due to twisting of knee particularly when knee is in mild flexion (less than $30^{\circ}$ ). 


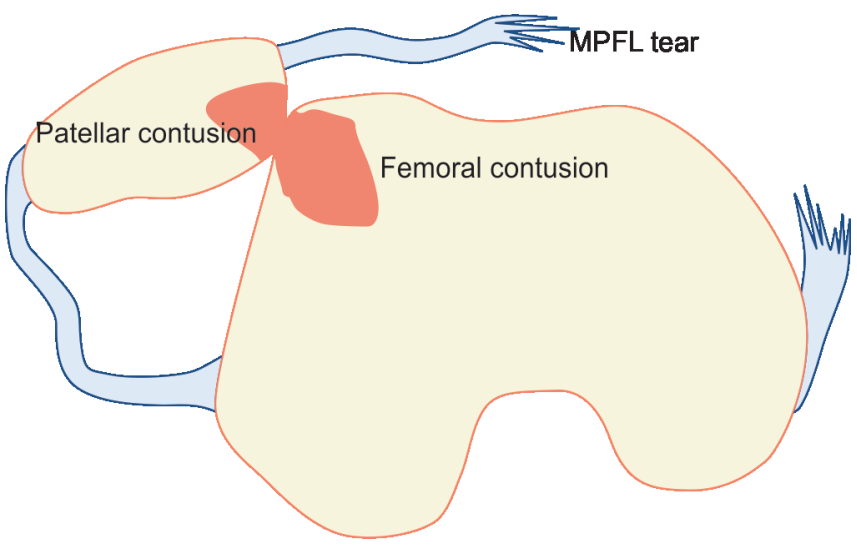

Fig. 1: Schematic image to depict the mechanism and injury pattern of TLPD

During the above-mentioned case, scenario patella undergoes a transient lateral displacement. During this dislocation phase, articular surfaces of either lateral femoral condyle or patella can get damaged by the shearing forces. Subsequently with reduction and extension at the knee joint, the patella relocates, but during that process, there occurs impaction of medial aspect of patella with the anterior aspect of lateral femoral condyle, which leads to the characteristic marrow edema pattern (Fig. 1). ${ }^{14,15}$ This study was conducted to determine the prevalence of various injury patterns in patients with TLPD on MRI.

\section{Materials and Methods}

This study was conducted after obtaining institutional ethical clearance. Thirty patients below 45 years of age with clinical history suggestive of patellar dislocation were enrolled in this study. Patients with prior history of infective/degenerative arthritis and patients in whom MRI was contraindicated were excluded from this study. After taking the informed, written consent of each patient, MRI was performed on Philips Achieva dStream 1.5 Tesla MRI using Sense Body Coil. This study was ethically conducted in accordance with the Declaration of Helsinki.

The following sequences were obtained: axial fat-suppressed proton density (FSPD), axial spin echo (SE) T, W, FSPD sagittal, and gradient echo (GRE) $\mathrm{T}_{2} \mathrm{~W}$ coronal.

Various MRI findings to be evaluated in this study are as follows:

- MR/MPFL injury,

- Contusion involving lateral femoral condyle,

- Contusion/osteochondral injury involving medial aspect of patella,

- Significant joint effusion,

- Patellar tilt,

- Patellar subluxation,

- Medial collateral ligament (MCL) injury, and

- Medial meniscal tear.

The MR/MPFL was assessed on axial sections and divided into three parts according to Palmer et al: ${ }^{16}$ at the level of its patellar insertion, at its mid-substance, and at its femoral origin (Fig. 2). The MR/MPFL was screened for partial or complete discontinuity with surrounding edema. Bone contusions involving lateral femoral condyle and medial aspect of patella were seen as areas

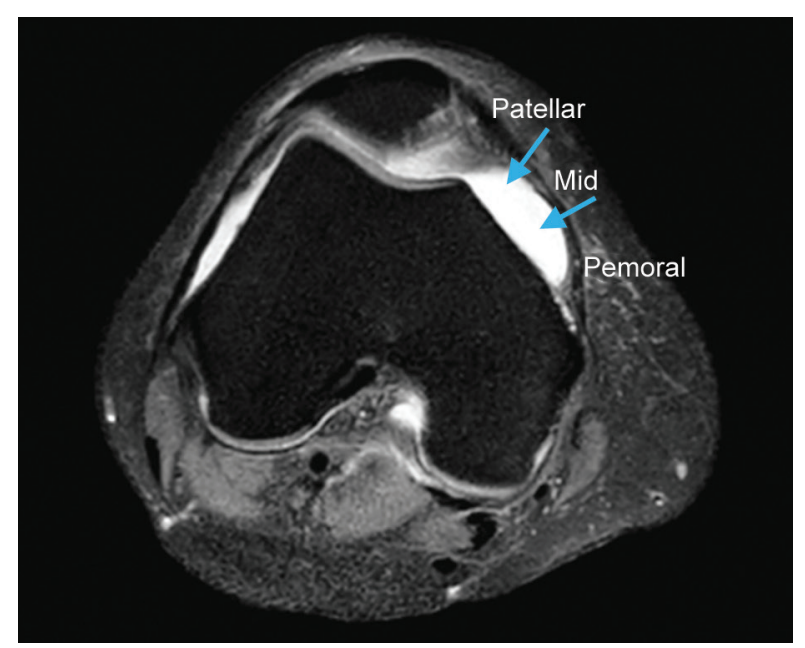

Fig. 2: Axial PD MRI image illustrating how medial patellofemoral ligament can be divided into three segments

of hypointensity in bone marrow on T1-weighted sequences and hyperintensity on PDFS sequences. Osteochondral injuries were identified as irregularity of the osteochondral surface of inferomedial patella. ${ }^{17}$ On sagittal images, joint effusion was considered significant if along the suprapatellar recess anteroposterior fluid depth was more than $4 \mathrm{~mm}$ in the midline and $10 \mathrm{~mm}$ lateral to the midline. ${ }^{18}$

Patellar tilt was estimated on axial MRI by comparing a line that crosses the anterior tips of the medial and lateral femoral condyles with a line drawn through the lateral patellar facet at the level of the midpatellar cartilage. The angle formed by the two lines is considered normal (absent for patellar tilt) if it opens laterally or abnormal (patellar tilt present) if the lines are parallel or the angle between them opens medially. ${ }^{19}$ Patellar subluxation is the lateralization of patellar apex with respect to the plane of trochlear apex. It was assessed on axial sections by deciding whether the patellar apex lay in the same parasagittal plane as the apex of the femoral trochlear groove. Medial meniscus was assessed for any tear. MCL was also assessed for any injury.

\section{Results}

Thirty patients (out of which 17 were females and 13 were males) with mean age of 21 years and age range of $10-45$ years with history of LPD were assessed. The majority of patients showed characteristic injury patterns (Table 1).

Of 30 patients, 25 (83.3\%) had MR/MPFL tear in general (Fig. 3).

- $50 \%(15$ of 30$)$ at its patellar insertion,

- $16.6 \%$ (5 of 30 ) at its mid-part, and

- $46.6 \%$ (14 of 30$)$ at its femoral insertion.

Eighty percentage ( 24 of 30 ) revealed contusions of the lateral femoral condyle (Fig. 4); 73.33\% (22 of 30) exhibited contusion/ osteochondral injury of medial aspect of patella (Fig. 5); $66.6 \%$ (20 of 30) showed significant effusion (Fig. 6). 56.6\% (17 of 30) exhibited patellar tilt (Fig. 7); $56.6 \%$ (17 of 30) showed patellar subluxation (Fig. 7); and $23.3 \%$ (7 of 30 ) revealed medial meniscal tear. Ten percentage ( 3 of 30 ) showed MCL injury (Fig. 8), and 6.6\% ( 2 of 30 ) showed intraarticular loose bodies. 
Table 1: Percentage frequency of various MRI injury patterns

\begin{tabular}{llll}
\hline Injury patterns & $\begin{array}{l}\text { Positive } \\
\text { cases }\end{array}$ & $\begin{array}{l}\text { Total number } \\
\text { of cases }\end{array}$ & Percentage \\
\hline MPFL/MR tear & 25 & 30 & 83.3 \\
Lateral femoral contusion & 24 & 30 & 80 \\
Medial patellar contusion/ & 22 & 30 & 73.3 \\
osteochondral injury & & & \\
Significant effusion & 20 & 30 & 66.6 \\
Patellar subluxation & 17 & 30 & 56.6 \\
Patellar tilt & 17 & 30 & 56.6 \\
Intraarticular loose body & 2 & 30 & 6.6 \\
MM tear & 7 & 30 & 23.3 \\
MCL injury & 3 & 30 & 10 \\
\hline
\end{tabular}

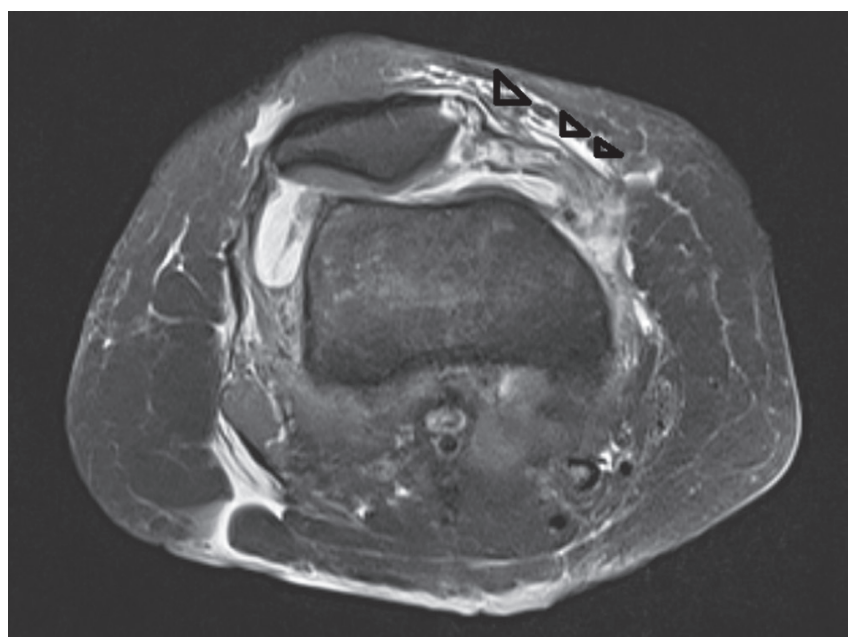

Fig. 3: Axial FSPD image: There is diffuse thickening and hyperintensity in MPFL consistent with tear of the ligament (black arrowheads)

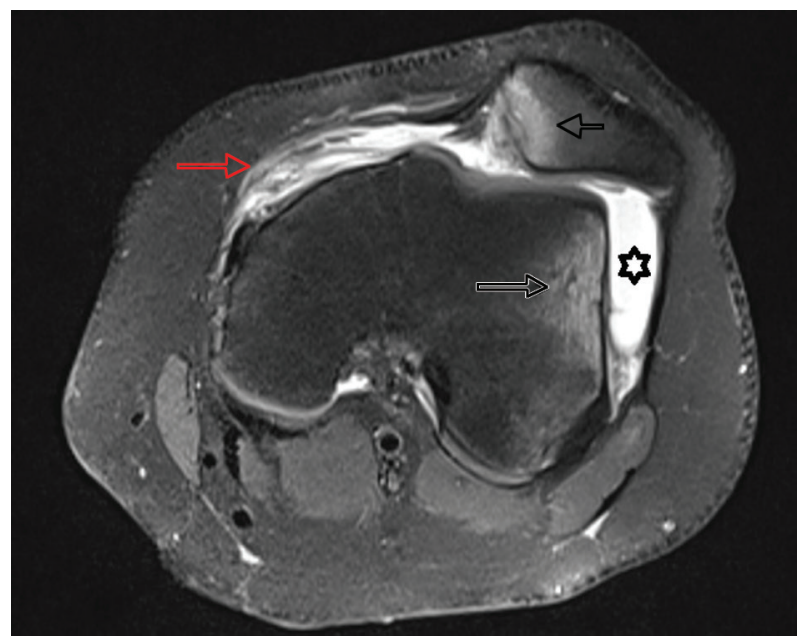

Fig. 4: Axial PD MRI image showing hyperintense signal in lateral femoral condyle and medial aspect of patella (black arrows)—suggestive of contusions. Associated tear of MPFL (red arrow) and effusion (asterisk) are also noted

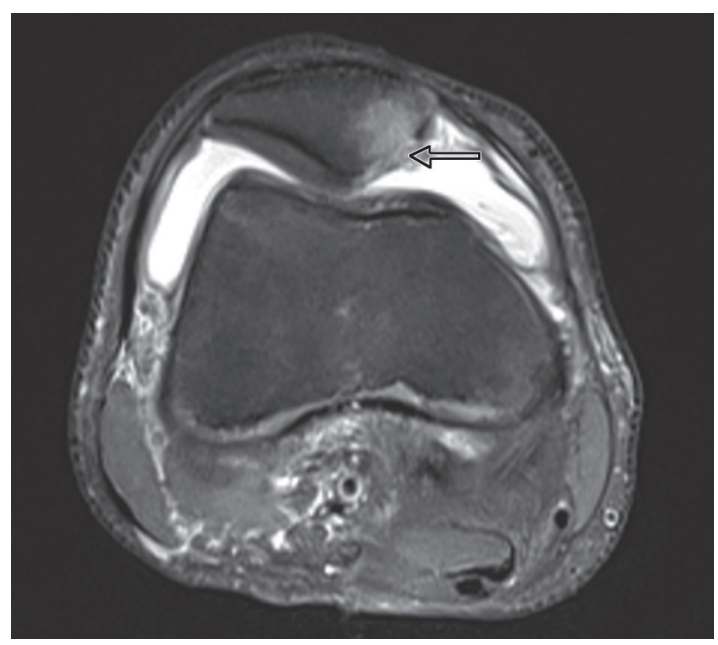

Fig. 5: Axial PD MRI image demonstrates osteochondral injury involving medial aspect of patella (black arrow)

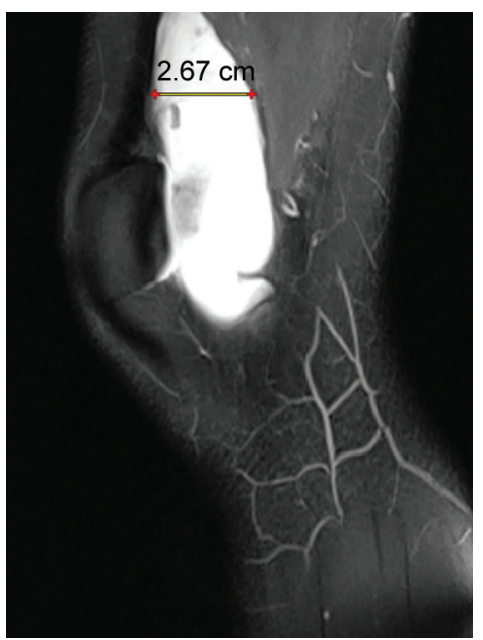

Fig. 6: Sagittal PD MRI image showing significant joint effusion in suprapatellar recess

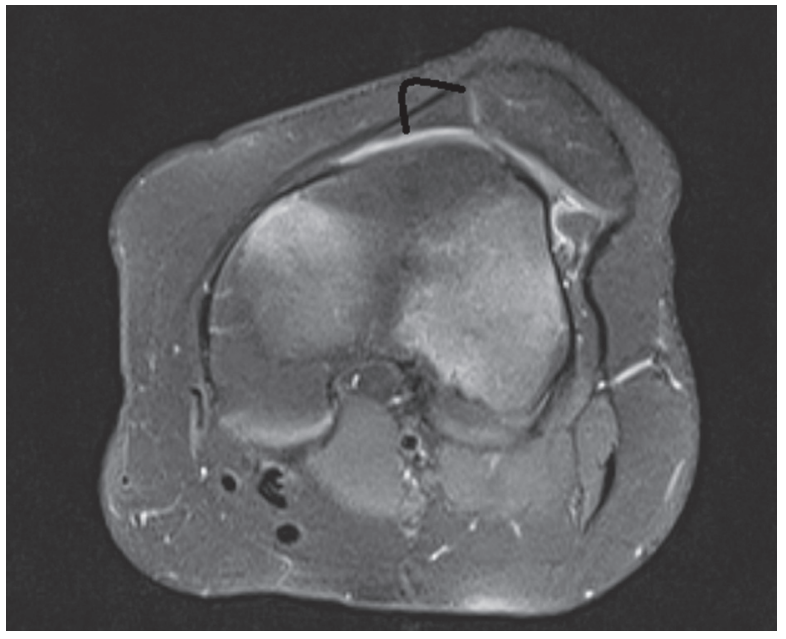

Fig. 7: Axial PD MRI image showing subluxated patella with positive patellar tilt. The arc in the image depicts the medial opening of angle formed between lateral patellar facet and anterior femoral condyles 


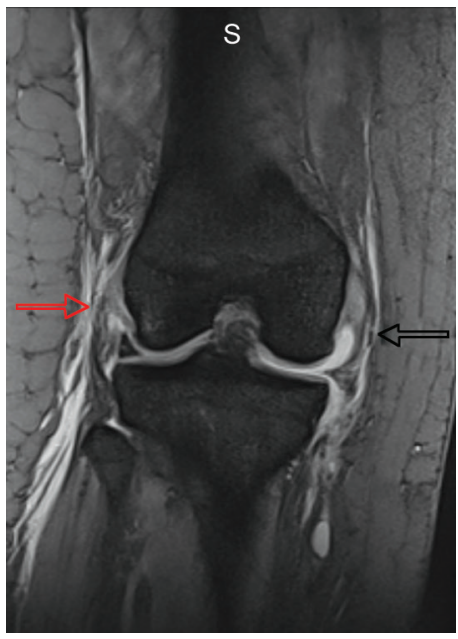

Fig. 8: Coronal T2 MED MRI image showing tear of MCL (black arrow). Incidental tear of lateral collateral ligament (LCL) (red arrow), effusion, and soft tissue edema are also noted

\section{Discussion}

TLPD is common in young adults involved in sports and dancerelated activities. Previously, plain radiograph was used to diagnose it, but many studies have concluded that radiographs have limited value to diagnose TLPD with diagnostic strength of as low as $32 \%$ in one of the studies. ${ }^{20}$ Therefore, MRI is the modality of choice to diagnose TLPD. Our study assessed the role of MRI in the detection of associated MRI findings seen in the case of patellar instability, which can indirectly suggest the diagnosis. Various associated MRI findings evaluated in this study were MR/MPFL injury, bone marrow edema/contusions involving lateral femoral condyle, bone marrow edema/contusions/osteochondral injuries involving medial aspect of patella, significant effusion, patellar tilt, patellar subluxation, $\mathrm{MCL}$ injury, and medial meniscal tear.

MR/MPFL is the main medial ligamentous stabilizer, and it is the most common injury associated with TLPD. Axial images are best for the evaluation of MR/MPFL. ${ }^{21}$ Of 30 patients, 25 (83.3\%) showed MR/MPFL tear in general. This is in concordance with study by Lance et al. ${ }^{22}$ and Spritzer et al. ${ }^{23}$ where they have shown that 80 to $90 \%$ of patellar dislocation shows concomitant MPFL tear.

The dislocated patella as it recovers its position clashes with the lateral femoral condyle. Hence, contusions in these locations are common. Of 30 cases, $24(80 \%)$ showed contusions of the lateral femoral condyle, and 22 (73.3\%) showed contusion/osteochondral injury of medial aspect of patella. This correlates with the study done by Elias et al. ${ }^{7}$ where they showed nearly similar results of 80 and $61 \%$ cases of patellar dislocation positive for lateral femoral condyle contusion and medial patellar contusion, respectively, while Quinn et al. ${ }^{24}$ in their study showed lateral femoral condyle contusion and medial patellar contusion in $100 \%$ cases.

Of 30 cases, 20 (66.6\%) showed significant effusion. We identified fewer cases with effusion, in comparison with previous studies by Lance et al., ${ }^{22}$ Spritzer et al., ${ }^{23}$ Kirsch et al., ${ }^{25}$ and Virolainen et al. ${ }^{26}$ in which effusion was seen in 95 to $100 \%$ of cases. This could be possibly due to strict criteria to diagnose clinically significant effusion used in our study. In our study on sagittal images, effusion was considered significant if along the suprapatellar recess anteroposterior fluid depth was more than
4 and $10 \mathrm{~mm}$ in the midline and lateral to midline, respectively. This could be further supported by nearly similar findings (55\%) by Elias et al. ${ }^{7}$ in which similar criteria for effusion were used.

Sometimes, the patella does not completely come back to its original position and remains subluxed. Of 30 cases, 17 (56.6\%) showed patellar subluxation. This is in concordance with studies of Virolainen et al. ${ }^{26}$ and Lance et al., ${ }^{22}$ in which patellar subluxation was seen in 64 and $50 \%$ cases, respectively. However, Kirsch et al. ${ }^{25}$ in their study showed patellar subluxation in $92 \%$ cases.

Of 30 patients, $3(10 \%)$ showed MCL injury. Elias et al. in their study also showed similar results with MCL injury seen in $11 \%$ of their cases. $^{7}$

\section{Conclusion}

Since LPD is a transient phenomenon, so we have to rely on secondary findings for its diagnosis. In this study, we have discussed the incidence of these characteristic injury patterns including contusions of the lateral femoral condyle, contusion/osteochondral injury of medial patella, significant effusion, patella subluxation, patellar tilt, MR/MPFL tear, which are the most common magnetic resonance injury patterns in TLPD. These findings are therefore essential to be recognized on routine MRI studies since prior history is not always present.

\section{ACKnOWLedgments}

We hereby acknowledge all patients for acting as valuable subjects for this study.

\section{Clinical Significance}

These findings can reliably help in diagnosing TLPD, and it is essential for the radiologist to be aware of these so as not to miss the diagnosis.

\section{References}

1. Fithian DC, Paxton EW, Stone ML, et al. Epidemiology and natural history of acute patellar dislocation. Am J Sports Med 2004;32(5): 1114-1121. DOI: 10.1177/0363546503260788.

2. Hawkins RJ, Bell RH, Anisette G. Acute patellar dislocations. The natural history. Am J Sports Med 1986;14(2):117-120. DOI: $10.1177 / 036354658601400204$.

3. Lewallen L, Mclntosh A, Dahm D. First-time patellofemoral dislocation: risk factors for recurrent instability. J Knee Surg 2015;28(4):303-309. DOI: 10.1055/s-0034-1398373.

4. Lewallen LW, Mclntosh AL, Dahm DL. Predictors of recurrent instability after acute patellofemoral dislocation in pediatric and adolescent patients. Am J Sports Med 2013;41(3):575-581. DOI: $10.1177 / 0363546512472873$.

5. Hinton RY, Sharma KM. Acute and recurrent patellar instability in the young athlete. Orthop Clin North Am 2003;34(3):385-396. DOI: 10.1016/s0030-5898(03)00033-6.

6. Earhart C, Patel DB, White EA, et al. Transient lateral patellar dislocation: review of imaging findings, patellofemoral anatomy, and treatment options. Emerg Radiol 2013;20(1):11-23. DOI: 10.1007/ s10140-012-1073-9.

7. Elias DA, White LM. Imaging of patellofemoral disorders. Clin Radiol 2004;59(7):543-557. DOI: 10.1016/j.crad.2004.01.004.

8. White BJ, Sherman OH. Patellofemoral instability. Bull NYU Hosp Jt Dis 2009;67(1):22-29. PMID: 19302054.

9. Kapur S, Wissman RD, Robertson M, et al. Acute knee dislocation: review of an elusive entity. Curr Probl Diagn Radiol 2009;38(6): 237-250. DOI: 10.1067/j.cpradiol.2008.06.001. 
10. Diederichs G, Issever AS, Scheffler S. MR imaging of patellar instability: injury patterns and assessment of risk factors. Radiographics 2010;30(4):961-981. DOI: 10.1148/rg.304095755.

11. Elias DA, White LM, Fithian DC. Acute transient lateral patellar dislocation at MR imaging: injury patterns of medial patellar softtissue restraints and osteochondral injuries of the inferomedial patella. Radiology 2002;225(3):736-743. DOI: 10.1148/radiol.2253011578.

12. Rhee SJ, Pavlou G, Oakley J, et al. Modern management of patellar instability. Int Orthop 2012;36(12):2447-2456. DOI: 10.1007/s00264-012-1669-4.

13. Askenberger M, Janarv PM, Finnbogason T, et al. Morphology and anatomic patellar instability risk factors in first-time traumatic lateral patellar dislocations: a prospective magnetic resonance imaging study in skeletally immature children. Am J Sports Med 2017;45(1):50-58. DOI: 10.1177/0363546516663498.

14. Sanders TG, Medynski MA, Feller JF, et al. Bone contusion patterns of the knee at MR imaging: footprint of the mechanism of injury. Radiographics 2000;20 Spec No:S135-S151. DOI: 10.1148/ radiographics.20.suppl_1.g00oc19s135.

15. Ballehr LO. Transient Lateral patellar dislocation. MRI Web Clinic, April 2013.

16. Palmer WE, Kuong SJ, Elmadbouh HM. MR imaging of myotendinous strain. AJR Am J Roentgenol 1999;173(3):703-709. DOI: 10.2214/ ajr.173.3.10470908.

17. Seeley MA, Knesek M, Vanderhave KL. Osteochondral injury after acute patellar dislocation in children and adolescents. J Pediatr Orthop 2013;33(5):511-518. DOI: 10.1097/BPO.0b013e318288b7a0.

18. Schweitzer ME, Falk $A$, Berthoty $D$, et al. Knee effusion: normal distribution of fluid. AJR Am J Roentgenol 1992;159(2):361-363. DOI: 10.2214/ajr.159.2.1632356.
19. Stanitski CL, Paletta GA Jr. Articular cartilage injury with acute patellar dislocation in adolescents. Arthroscopic and radiographic correlation. Am J Sports Med 1998;26(1):52-55. DOI: 10.1177/03635465980260012501.

20. Koskinen SK, Kujala UM. Patellofemoral relationships and distal insertion of the vastus medialis muscle: a magnetic resonance imaging study in nonsymptomatic subjects and in patients with patellar dislocation. Arthroscopy 1992;8(4):465-468. DOI: 10.1016/0749-8063(92)90009-z.

21. Dirim B, Haghighi $P$, Trudell D, et al. Medial patellofemoral ligament: cadaveric investigation of anatomy with MRI, MR arthrography, and histologic correlation. AJR 2008;191(2):490-498. DOI: 10.2214/ AJR.07.3590.

22. Lance $E$, Deutsch AL, Mink JH. Prior lateral patellar dislocation: $M R$ imaging findings. Radiology 1993;189(3):905-907. DOI: 10.1148/ radiology.189.3.8234724.

23. Spritzer CE, Courneya DL, Burk DLJ, et al. Medial retinacular complex injury in acute patellar dislocation: MR findings and surgical implications. AJR Am J Roentgenol 1997;168(1):117-122. DOI: 10.2214/ ajr.168.1.8976933.

24. Quinn SF, Brown TR, Demlow TA. MR imaging of patellar retinacular ligament injuries. J Magn Reson Imaging 1993;3(6):843-847. DOI: 10.1002/jmri.1880030607.

25. Kirsch MD, Fitzgerald SW, Friedman H, et al. Transient lateral patellar dislocation: diagnosis with MR imaging. AJR Am J Roentgenol 1993;161(1):109-113. DOI: 10.2214/ajr.161.1.8517287.

26. Virolainen $\mathrm{H}$, Visuri $\mathrm{T}$, Kuusela $\mathrm{T}$. Acute dislocation of the patella: MR findings. Radiology 1993;189(1):243-246. DOI: 10.1148/ radiology.189.1.8372201. 